\title{
聚苯胺包覆蛋白石页岩/硫复合材料的制备及其电化学性能
}

\author{
柴二亚 ${ }^{1}$, 潘俊安 ${ }^{1}$, 袁国龙 ${ }^{1}$, 程 豪 $^{1}$, 安 峰 $^{1}$, 谢淑红 ${ }^{2}$
}

(1. 湘潭大学 材料科学与工程学院, 薄膜材料及器件湖南省重点实验室, 湘潭 $411105 ; 2$. 湘潭大学低维材料及 其应用技术教育部重点实验室, 湘潭 411105)

摘 要: 以蛋白石页岩为载硫体, 通过化学沉积法制备蛋白石页岩/硫复合材料, 再利用化学氧化聚合法在其表面包 覆一层聚苯胺, 制备出一种新型的蛋白石页岩/硫-聚苯胺复合材料, 作为锂硫电池的正极材料。SEM、TEM 和 BET 等测试结果表明蛋白石页岩呈层状多孔结构, 小尺寸硫在材料内分布均匀, 聚苯胺包覆的厚度约为 $400 \mathrm{~nm}$ 。电化学性 能测试表明, 蛋白石页岩/硫-聚苯胺正极活化后放电比容量最高达到 $1164.93 \mathrm{mAh} / \mathrm{g}$, 在 $0.5 C(1.0 C=1675 \mathrm{~mA} / \mathrm{g})$ 倍 率下, 循环 300 次后放电比容量为 $539.30 \mathrm{mAh} / \mathrm{g}$, 库伦效率始终保持在 $95 \%$ 以上, 说明蛋白石页岩具有良好的吸附 性，同时导电聚苯胺包覆层具有双效固硫的作用，有利于吸附多硫化物和抑制穿梭效应。

关 键 词: 蛋白石页岩; 聚苯胺; 正极材料; 锂硫电池

中图分类号: 0646 文献标识码: A

\section{Preparation and Electrochemical Property of Polyaniline Coated Opal Shale/Sulfur Composite}

\author{
CHAI Er-Ya ${ }^{1}$, PAN Jun-An ${ }^{1}$, YUAN Guo-Long ${ }^{1}$, CHENG Hao ${ }^{1}$, AN Feng ${ }^{1}$, XIE Shu-Hong ${ }^{2}$ \\ (1. Hunan Provincial Key Laboratory of Thin Film Materials and Devices, School of Materials Science and Engineering, \\ Xiangtan University, Xiangtan 411105, China; 2. Key Laboratory of Low Dimensional Materials and Application Technology \\ of Ministry of Education, Xiangtan University, Xiangtan 411105, China)
}

\begin{abstract}
Opal shale/S composite was prepared by loading sulfur on opal shale via solution-based reaction- deposition method, and then polyaniline (PANI) was covered on the as-prepared opal shale/S composite by means of chemical oxidative polymerization method, which was used as cathode material of lithium-sulfur battery. Scanning electron microscope (SEM), transmission electron microscope (TEM), and Brunauer-Emmett-Teller (BET) characterization were conducted, and the results indicated that opal shale had a layered porous structure. As-prepared sulfur with small size was distributed uniformly in the composite. The PANI with around thickness of $400 \mathrm{~nm}$ was coated on the surface of the opal shale/S composite. Constant current charge-discharge tests showed that opal shale/S-PANI cathode obtained a maximum discharge specific capacity of $1164.93 \mathrm{mAh} / \mathrm{g}$ after activation and retained a discharge specific capacity of $539.30 \mathrm{mAh} / \mathrm{g}$ after 300 cycles at the current rate of $0.5 C(1.0 C=1675 \mathrm{~mA} / \mathrm{g})$. The coulombic efficiency of opal shale/S-PANI cathode always maintained more than $95 \%$, indicating that opal shale with excellent adsorption properties and conductive PANI coating had double fixation effect for sulfur, which was beneficial to adsorb polysulfide and inhibit the shuttle effect.
\end{abstract}

Key words: opal shale; polyaniline; cathode material; lithium-sulfur battery

收稿日期：2017-01-11; 收到修改稿日期：2017-02-28

基金项目: 国家自然科学基金(11472236, 11627801); 湖南省科学技术厅科技计划(2015JC3092)

National Natural Science Foundation of China (11472236, 11627801); Program for Science and Technology of Hunan Province (2015JC3092)

作者简介: 柴二亚(1991-)，女，硕士研究生. E-mail: 18295950394@163.com

通讯作者：谢淑红，教授. E-mail: shxie@xtu.edu.cn 
相比于传统锂离子电池, 锂硫电池具有较高的质 量能量密度 $(2600 \mathrm{Wh} / \mathrm{kg})$ 和体积能量密度 $(2800 \mathrm{Wh} / \mathrm{L})$, 并且单质硫价格便宜、资源丰富, 从而成为近年来 的研究热点之一 ${ }^{[1-3]}$ 。但是, 锂硫电池商业化应用仍 面临许多挑战, 主要包括: (1)反应物 $\left(\mathrm{S}_{8}\right)^{[4]}$ 和放电终 产物 $\left(\mathrm{Li}_{2} \mathrm{~S} \text { 和 } \mathrm{Li}_{2} \mathrm{~S}_{2}\right)^{[5]}$ 导电性差, 导致电极材料活化 困难; (2)硫电极在充放电过程中体积发生变化 ${ }^{[6]}$, 加速电极的粉碎和破坏; (3)反应中间产物多硫化 物 $\left(\mathrm{Li}_{2} \mathrm{~S}_{x}, 2<x<8\right)$ 易溶于有机电解液, 导致活性物质 流失及 “穿梭效应” ${ }^{[7]}$, 从而严重影响电池的电化 学性能。

近年来, 锂硫电池研究者利用 $\mathrm{SiO}_{2}$ 与 $\mathrm{S}$ 复合来 抑制多硫化物的溶解和扩散, 取得了一定成果。 $\mathrm{Nazar}^{[8]}$ 和 $\mathrm{Kim}^{[9]}$ 等发现将 $\mathrm{SiO}_{2}$ 纳米颗粒加入到硫正 极复合材料中, 可以有效地改善电池的循环性能, 这是由于 $\mathrm{SiO}_{2}$ 纳米颗粒表面的 $\mathrm{Si}-\mathrm{O}$ 基团带正电荷, 有利于吸附极性多硫化物, 抑制 “穿梭效应”。蛋白 石页岩 ${ }^{[10-12]}$ 是一种 $\mathrm{SiO}_{2}$ 含量超过 $80 \%$ 的天然矿物, 具有微米级粒径、纳米级孔, 其储量丰富、比重小、 结构疏松多孔、比表面积大、吸附性强, 具有较好 的离子交换能力和亲硫性。随着锂硫电池研究工作 的深入, 人们发现包覆结构可以提高电极材料的导 电性，缓解体积形变，同时还可以抑制“穿梭效应”, 有利于改善锂硫电池的电化学性能 ${ }^{[13-17]}$ 。其中, 聚 苯胺 ${ }^{[16-18]}$ 是一种最常见的导电聚合物, 具有特殊的 官能团和独特的链结构, 可与硫或多硫化物发生化 学作用, 有效地束缚多硫化物, 同时还具有导电性 好、成本低、易合成和不溶于电解液等优点, 常被 用于包覆硫电极以改善锂硫电池的性能。

因此, 本工作以蛋白石页岩为载硫体, 在其表 面包覆一层聚苯胺, 利用蛋白石页岩和聚苯胺的协 同作用, 双效抑制多硫化物的溶解和扩散, 从而提 高锂硫电池的电化学性能。

\section{1 实验方法}

\section{1 正极材料的制备}

\subsection{1 蛋白石页岩/硫复合材料的制备}

将 $0.5 \mathrm{~g}$ 蛋白石页岩加入到 $10 \mathrm{~mL}$ 蒸馏水中, 再 加入 $3.875 \mathrm{~g}$ 五水合硫代硫酸钠, 超声震荡 $30 \mathrm{~min}$; 然后向混合液逐滴加入适量盐酸, 磁力搅拌 $12 \mathrm{~h}$ 后 用蒸馏水洗涤至 $\mathrm{pH}=7$ 。最后经 $60^{\circ} \mathrm{C}$ 真空干燥 $12 \mathrm{~h}$, 得到蛋白石页岩/硫复合材料。

\subsection{2 蛋白石页岩/硫-聚苯胺复合材料的制备}

将 $1 \mathrm{~g}$ 蛋白石页岩/硫复合材料加入到 $10 \mathrm{~mL}$ 乙
醇中，超声分散均匀; 抽取 $0.5 \mathrm{~mL}$ 苯胺单体滴加到 $10 \mathrm{~mL}$ 盐酸中, 搅拌 $30 \mathrm{~min}$ 后与蛋白石页岩/硫溶液 混合, 然后置于冰水浴中, 将氯化铁溶液缓慢滴加 到混合液中，持续搅拌 $12 \mathrm{~h}$ 。经抽滤、洗涤, $60^{\circ} \mathrm{C}$ 真 空干燥 $24 \mathrm{~h}$, 制得蛋白石页岩/硫-聚苯胺复合材料。

\section{2 材料表征}

采用 Minflex 型 X 射线衍射仪(XRD)对样品进 行物相分析, 辐射源为 $\mathrm{CuK \alpha}$, 扫描速度为 $5 \%$ min。 采用 S-4800 型扫描电子显微镜(SEM)和 Tecnai G2 F20 型透射电子显微镜(TEM)对样品进行形貌表征。 采用 JW-BK122W 型比表面积测试仪(BET)测试样 品的比表面积和孔径分布。采用 Q50 型热重分析仪 (TGA)对样品进行热重分析。

\section{3 电池组装和性能测试}

将制好的复合材料、乙炔黑和聚偏氟乙烯 (PVDF)以 7:2:1 的质量比混合, 加入适量 $\mathrm{N}$-甲基 吡咯烷酮(NMP), 磁力搅拌 $12 \mathrm{~h}$ 。将得到的浆料均 匀涂在铝箔上, 在 $60^{\circ} \mathrm{C}$ 真空干燥 $12 \mathrm{~h}$, 裁成 $\phi 14 \mathrm{~mm}$ 的极片。制得的正极片中复合材料、乙炔黑和 PVDF 的负载量分别约为 1.53、0.44 和 $0.22 \mathrm{mg} / \mathrm{cm}^{2}$, 并以 锂片为负极, Celgard 2400 为隔膜, $1 \mathrm{~mol} / \mathrm{L} \mathrm{LiTFSI} /$ $\operatorname{DOL}-\operatorname{DME}(1: 1, V: V)$ 为电解液, 在氩气气氛的手 套箱(Mikrouna)中组装 2016 型扣式电池。使用电池 测试系统(BTS-5V3A) 在室温下进行恒流充放电性 能测试, 电压范围为 $1.0 \sim 3.0 \mathrm{~V}\left(v s . \mathrm{Li} / \mathrm{Li}^{+}\right)$。使用电化 学工作站(CHI660D)进行循环伏安测试(CV: 扫描电 压范围为 $1.0 \sim 3.0 \mathrm{~V}$, 扫描速率为 $0.1 \mathrm{mV} / \mathrm{s}$ ) 和交流阻 抗测试(EIS: 测试频率范围为 $0.1 \mathrm{~Hz} 1 \mathrm{MHz}$ )。

\section{2 结果与讨论}

\section{1 样品的结构与形貌}

图 1 为硫、蛋白石页岩、蛋白石页岩/硫和蛋白 石页岩/硫-聚苯胺的 XRD 图谱。由图 1 可知, 硫属 于 Fddd 型斜方晶系结构, 衍射峰尖锐, 说明其结晶 性好。蛋白石页岩主要由正方晶系的方石英(PDF\#820512)和六方晶系的 $\alpha$-石英(PDF\#89-1961)组成。两 种复合材料均具有硫和二氧化硅的特征峰, 且峰强 明显低于单质硫。相比于包覆前, 包覆聚苯胺后复 合材料的衍射强度略有降低。

图 2 为蛋白石页岩、蛋白石页岩/硫和蛋白石页 岩/硫-聚苯胺的 SEM 和 TEM 照片。图 2(a)显示蛋 白石页岩具有层状结构, 这种结构有利于硫的附着, 但蛋白石页岩粉末大小不均匀, 尺寸分布在 $0.5 \sim$ $6 \mu \mathrm{m}$ 范围内。图 2(b)显示片层状结构的蛋白石页岩 


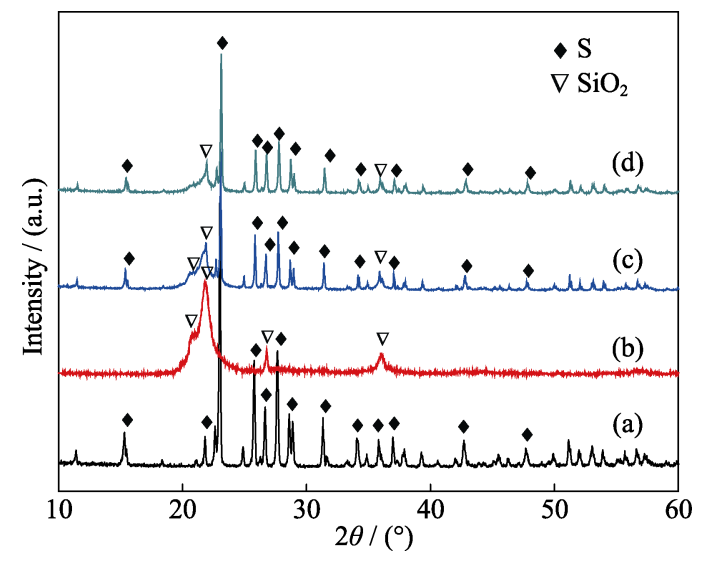

图 1 硫(a)、蛋白石页岩 $(b)$ 、蛋白石页岩/硫(c)和蛋白石页 岩/硫-聚苯胺(d)的 XRD 图谱

Fig. 1 XRD patterns of S (a), opal shale (b), opal shale/S (c) and opal shale/S-PANI (d)
由 50 150 $\mathrm{nm}$ 的晶粒构成。图 2(c)显示蛋白石页岩 经化学法注硫后粉末平均粒径有所减小, 尺寸分布 在 $0.5 \sim 4 \mu \mathrm{m}$ 范围内, 有硫附着在其表面。图 2(d)证 实化学法制备的蛋白石页岩/硫复合材料中硫颗粒 的尺寸较小, 大小在 20 40 $\mathrm{nm}$, 且分散性较好。图 2(e)显示包覆聚苯胺后蛋白石页岩/硫的平均粉末 粒径增大, 尺寸分布在 1 9 $\mu \mathrm{m}$ 范围内。图 2(f)显示 蛋白石页岩/硫表面包覆的聚苯胺厚度约为 $400 \mathrm{~nm}$ 。

\section{2 比表面积和孔径分析}

图 3(a)为蛋白石页岩、蛋白石页岩/硫和蛋白石 页岩/硫-聚苯胺的氮气等温吸脱附曲线, 均属于 IUPAC 分类中的 IV 型、H3 型滞后环 ${ }^{[19]}$ 。当相对压 力 $\left(P / P_{0}\right)$ 较小时, 吸附量缓慢增加, 曲线呈上升趋势; 当相对压力在 $0.8 \sim 1.0$ 时, 吸附量大幅增加, 这是因
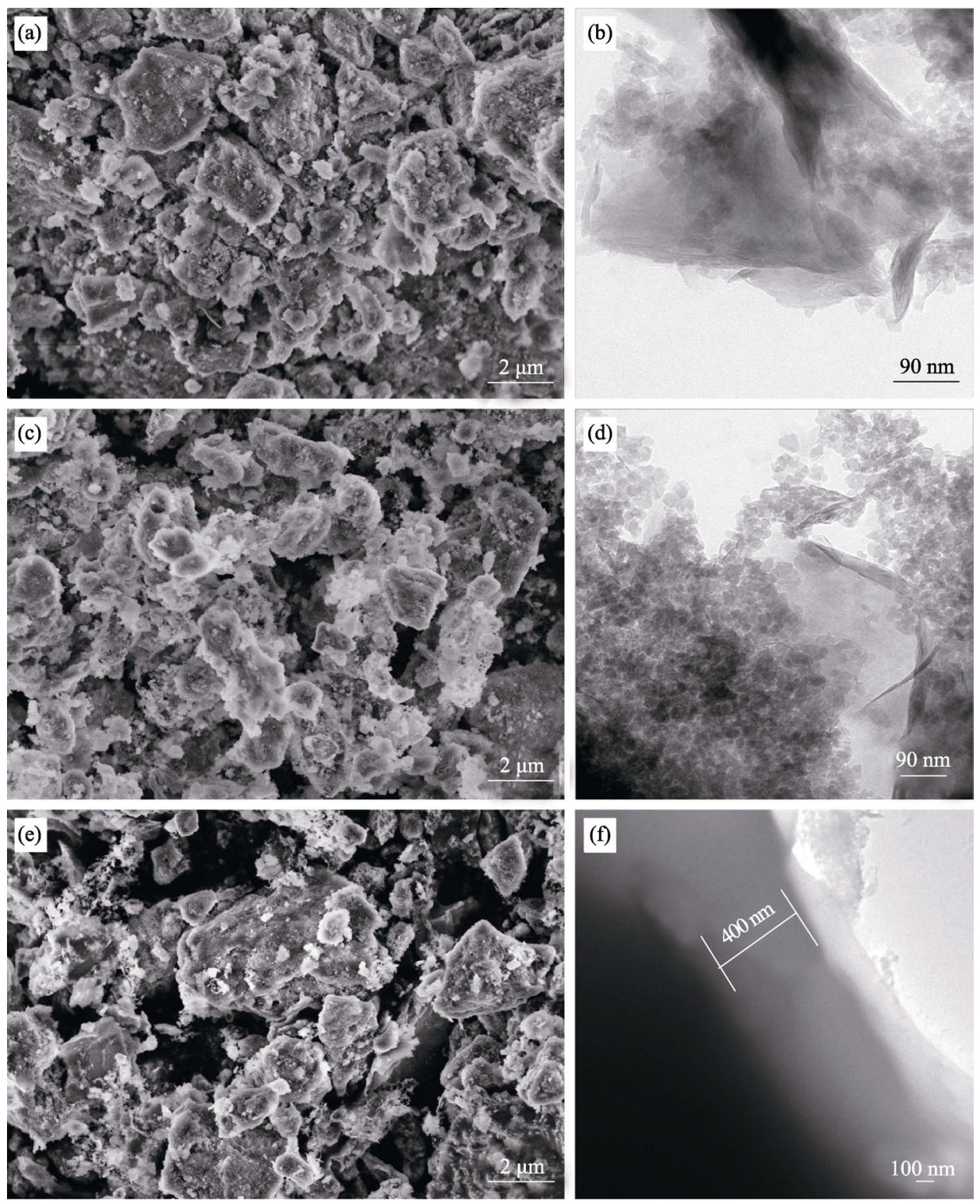

图 2 蛋白石页岩 $(a, b)$, 蛋白石页岩/硫 $(c, d)$, 蛋白石页岩/硫-聚苯胺 $(e, f)$ 的 SEM 和 TEM 照片

Fig. 2 SEM and TEM morphologies of opal shale (a,b), opal shale/S (c, d), and opal shale/S-PANI (e, f), respectively 

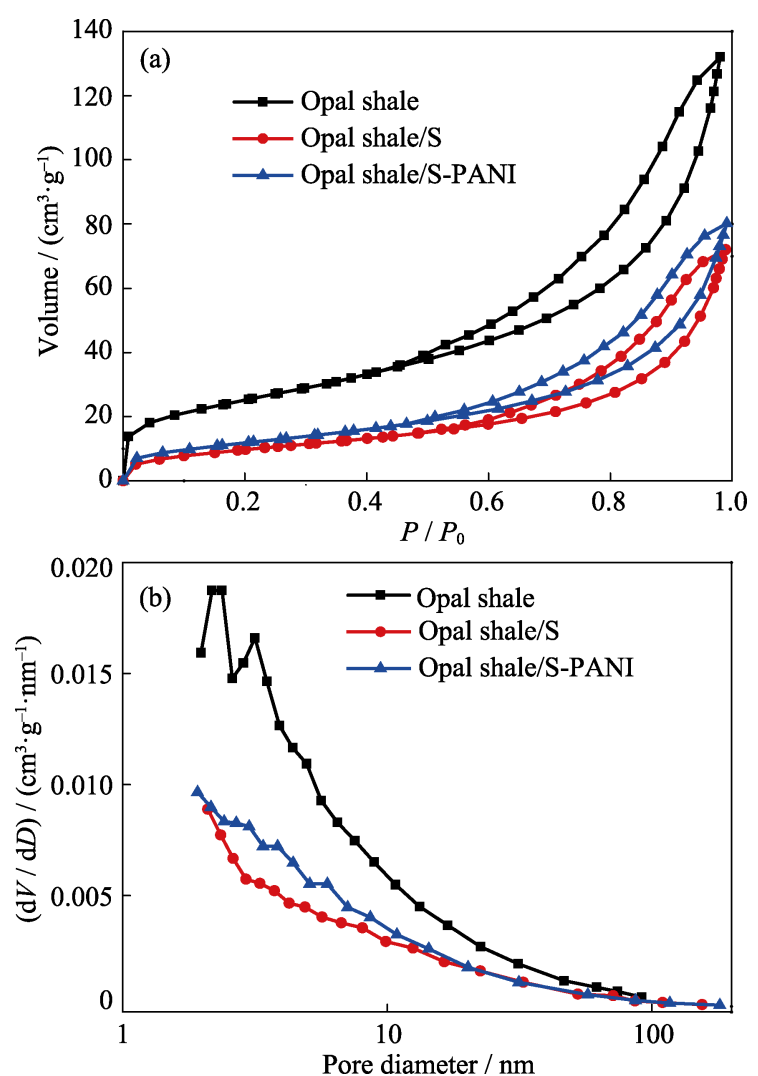

图 3 蛋白石页岩、蛋白石页岩/硫和蛋白石页岩/硫-聚苯 胺的氮气等温吸脱附图(a)和孔径分布图(b)

Fig. 3 Nitrogen adsorption-desorption isotherms (a) and BJH pore size distribution (b) of opal shale, opal shale/S and opal shale/S-PANI

为材料中存在大量的介孔, 在高压区发生毛细凝聚 使得氮气吸附量增加 ${ }^{[20]}$ 。蛋白石页岩的比表面积和 孔容分别为 $90.78 \mathrm{~m}^{2} / \mathrm{g}$ 和 $0.20 \mathrm{~cm}^{3} / \mathrm{g}$, 蛋白石页岩/硫 的比表面积和孔容分别减小为 $33.93 \mathrm{~m}^{2} / \mathrm{g}$ 和 $0.11 \mathrm{~cm}^{3} / \mathrm{g}$, 表明部分硫进入蛋白石页岩的孔隙中，使得蛋白石 页岩的孔隙率减小。蛋白石页岩/硫-聚苯胺的比表 面积和孔容分别为 $44.02 \mathrm{~m}^{2} / \mathrm{g}$ 和 $0.12 \mathrm{~cm}^{3} / \mathrm{g}$, 相比于 包覆前略有增大, 这是因为化学氧化法制得的聚苯 胺结构具有多孔性, 且比表面积较大, 可适当增大 材料孔隙率。

图 3(b)为样品对应的孔径分布图, 可以看出蛋 白石页岩的孔径主要为 $2 \sim 5 \mathrm{~nm}$ 的介孔, 注硫后平 均孔径减小, 聚苯胺包覆后平均孔径略有增大。

\section{3 蛋白石页岩/硫一聚苯胺的热重分析}

图 4 为蛋白石页岩/硫-聚苯胺在氮气气氛中的 热重分析曲线, 温度测试范围为 $50 \sim 700^{\circ} \mathrm{C}$, 升温速 率为 $10^{\circ} \mathrm{C} / \mathrm{min}$ 。由图 4 可知, 蛋白石页岩/硫-聚苯 胺的质量损失主要分为两个阶段, 当温度在 150 $280^{\circ} \mathrm{C}$ 范围时, 由于硫的蒸发引起失重, 对应硫的质 量分数为 $46.6 \%$; 当温度在 $280 \sim 600^{\circ} \mathrm{C}$ 范围时, 由于聚 苯胺的分解引起失重, 对应聚苯胺的质量分数为 $6.7 \%$ 。

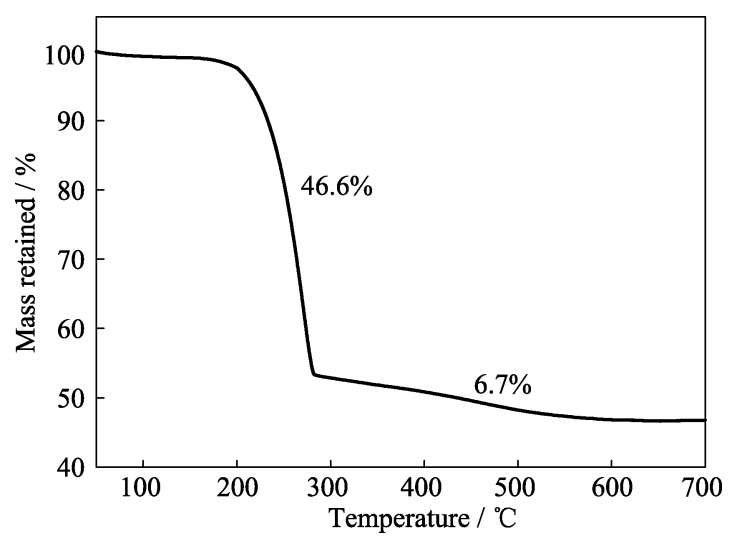

图 4 蛋白石页岩/硫-聚苯胺的热重分析曲线

Fig. 4 Thermo gravimetric analysis (TGA) curve of opal shale/S-PANI

\section{4 充放电性能测试}

图 5(a)为 $0.5 C$ 倍率下蛋白石页岩/硫和蛋白石 页岩/硫-聚苯胺循环性能图, 由图可知, 蛋白石页 岩/硫的首次放电比容量为 $832.39 \mathrm{mAh} / \mathrm{g}$, 循环 300 次后衰减为 $373.48 \mathrm{mAh} / \mathrm{g}$, 容量保持率为 $44.87 \%$, 库伦效率保持在 95\%左右，表明蛋白石页岩对多硫
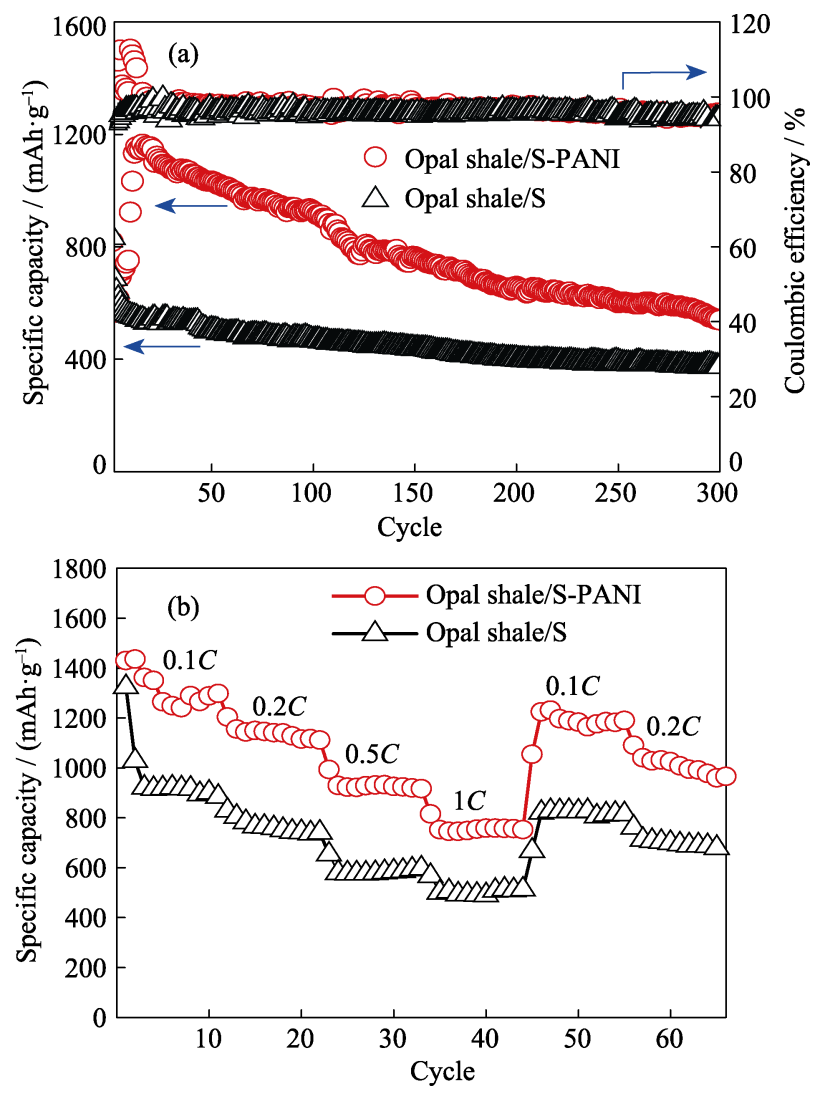

图 5 蛋白石页岩/硫和蛋白石页岩/硫-聚苯胺在 $0.5 C$ 倍率 下的循环性能图(a)和倍率性能图(b)

Fig. 5 (a) Cycling performance and the corresponding coulombic efficiency at $0.5 \mathrm{C}$ rate; (b) rate capability under different discharge rate of the opal shale/S and opal shale/S- PANI 
化物有一定的吸附作用, 有利于提高电池的循环性 能。蛋白石页岩/硫-聚苯胺在循环初始阶段, 由于 聚苯胺的包覆, 一些深埋的硫需要较长时间与电解 液接触才能逐渐电化学活化 ${ }^{[21]}$, 放电比容量呈增大 趋势, 经过 16 次循环活化后, 放电比容量达到最大 为 $1164.93 \mathrm{mAh} / \mathrm{g}$; 随后放电比容量逐渐减小, 循环 300 次后衰减至 $539.30 \mathrm{mAh} / \mathrm{g}$, 容量保持率为 $46.29 \%$, 库伦效率始终保持在 $95 \%$ 以上。这是因为 聚苯胺包覆既能有效地抑制多硫化物的溶解和扩散, 增强材料的导电性, 又能在一定程度上缓解充放电 过程中硫正极产生的结构应力, 从而可以进一步提 高电池的性能。

图 5(b)为蛋白石页岩/硫和蛋白石页岩/硫-聚苯 胺的倍率性能图。在 $0.1 C$ 倍率下, 循环 11 次后蛋白 石页岩/硫-聚苯胺的放电比容量保持在 $1298.63 \mathrm{mAh} / \mathrm{g}$, 倍率增大至 $1 C$ 时, 放电比容量降至 $752.41 \mathrm{mAh} / \mathrm{g}$, 而当倍率回复至 $0.1 C$ 时, 放电比容量恢复至 $1224.45 \mathrm{mAh} / \mathrm{g}$ 。在相同的倍率下, 蛋白石页岩/硫对应 的放电比容量分别为 $884.67 、 500.36$ 和 $820.05 \mathrm{mAh} / \mathrm{g}$ 。 结果表明蛋白石页岩/硫和蛋白石页岩/硫-聚苯胺 均具有较好的倍率性能, 且包覆聚苯胺后的材料倍 率性能更加优异。

\section{5 循环伏安特性}

图 6 为蛋白石页岩/硫-聚苯胺的循环伏安曲线, 如图所示, 在 $2.25 \mathrm{~V}$ 和 $2.00 \mathrm{~V}$ 左右出现 2 个还原峰, 分别对应单质硫还原为高阶多硫化锂 $\left(\mathrm{Li}_{2} \mathrm{~S}_{x}, 4<x<8\right)$, 及高阶多硫化物进一步还原为低阶多硫化锂 $\left(\mathrm{Li}_{2} \mathrm{~S}_{x}\right.$, $2<x<4)$ 和硫化锂 $\left(\mathrm{Li}_{2} \mathrm{~S}\right)$ 的过程。在 $2.50 \mathrm{~V}$ 左右出现 1 个氧化峰, 对应 $\mathrm{Li}_{2} \mathrm{~S}$ 和 $\mathrm{Li}_{2} \mathrm{~S}_{2}$ 氧化生成单质硫的过 程。对比发现, 循环 300 次后, 因为极化的原因氧化 峰和还原峰分别向低电位和高电位移动, 但电位移 动不大, 表明电极材料具有较好的循环可逆性。

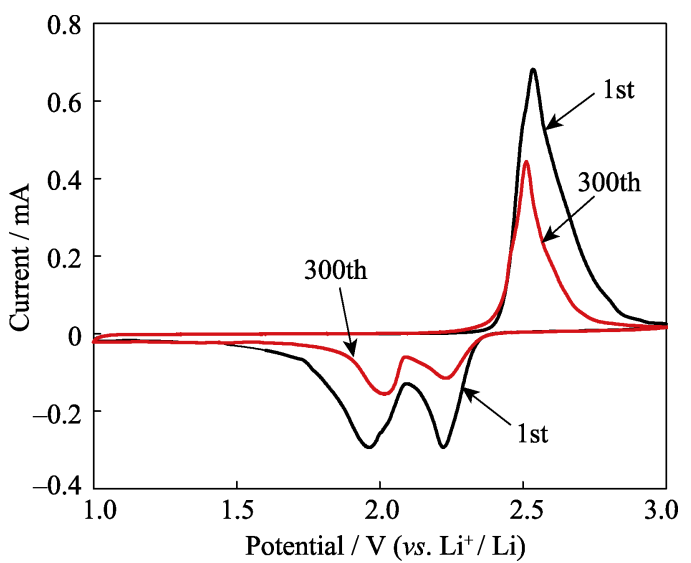

图 6 蛋白石页岩/硫-聚苯胺的循环伏安曲线

Fig. 6 Cyclic voltammetry curves of opal shale/S-PANI after the first cycle and 300th cycle at $0.5 \mathrm{C}$ rate

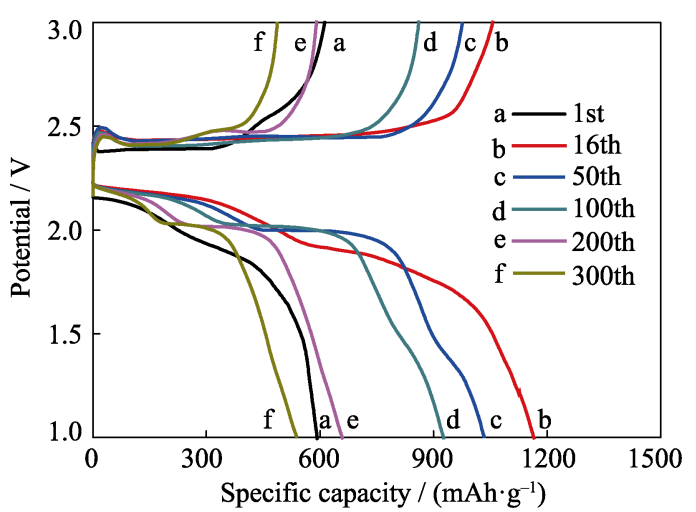

图 $70.5 C$ 倍率下蛋白石页岩/硫-聚苯胺的充放电曲线图 Fig. 7 Galvanostatic discharge-charge profiles of opal shale/ $\mathrm{S}-\mathrm{PANI}$ at $0.5 \mathrm{C}$ rate

图 7 为蛋白石页岩/硫-聚苯胺在 $0.5 C$ 倍率下分 别循环 $1 、 16 、 50 、 100 、 200 、 300$ 次的充放电曲线, 图中出现 2 个硫电极典型的放电平台, 与图 6 的循 环伏安测试结果一致, 表明硫和锂的反应是个多步 反应过程。循环初始阶段放电电压平台不明显, 循 环 16 次后平台逐渐稳定, 这是由于循环过程中电极 材料与电解液接触增多, 电极材料逐渐被活化造成 的。且材料的可逆性较好, 循环 300 次后, 电压平台 仍然明显且稳定。

\section{6 电化学交流阻抗测试}

图 8 为蛋白石页岩/硫-聚苯胺循环 1 次和 300 次的交流阻抗图。阻抗谱由高频区和中频区的 2 个 半圆组成, 高频区的半圆对应电极的接触电阻 ${ }^{[22]}$, 中频区的半圆对应电荷转移电阻 ${ }^{[23]}$ 。循环 300 次后, 电极的接触电阻略有减小, 这是因为循环过程中电 极材料逐渐被电解液润湿，电极材料活化完全，使 得接触电阻减小; 而电极的电荷转移电阻增大, 这 是因为在循环过程中, 电极材料的表面逐渐生成了 不溶于电解液的绝缘放电产物 $\mathrm{Li}_{2} \mathrm{~S}$ 和 $\mathrm{Li}_{2} \mathrm{~S}_{2}$, 导致电 荷转移阻抗增大。

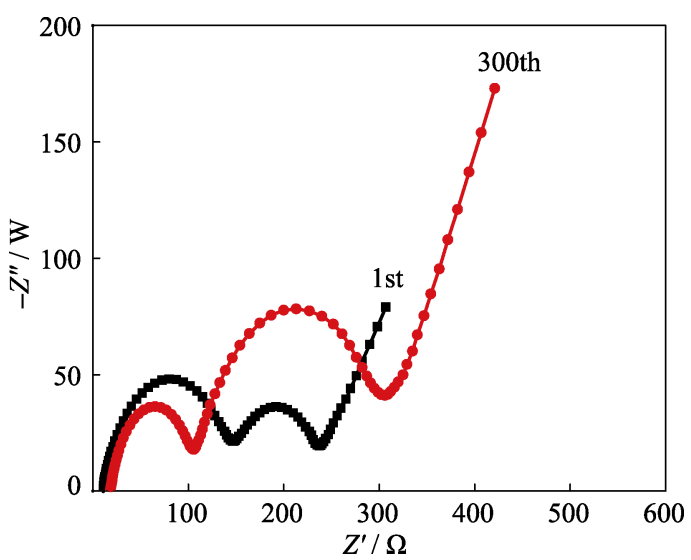

图 8 蛋白石页岩/硫-聚苯胺的交流阻抗图

Fig. 8 Electrochemical impedance spectroscopy of opal shale/SPANI after the first cycle and 300 th cycle at $0.5 \mathrm{C}$ rate 


\section{3 结论}

以蛋白石页岩为载硫体, 制备出具有包覆结构 的蛋白石页岩/硫-聚苯胺复合材料。实验结果表明: 包覆聚苯胺后的蛋白石页岩/硫复合材料具有优异 的电化学性能, 其放电比容量较高, 倍率性能较好, 库伦效率保持在 $95 \%$ 以上。在 $0.5 C$ 倍率下, 蛋白石 页岩/硫-聚苯胺经过活化之后, 放电比容量可达 $1164.93 \mathrm{mAh} / \mathrm{g}$, 循环 300 次后容量保持在 $539.30 \mathrm{mAh} / \mathrm{g}$, 远高于蛋白石页岩/硫相对应的放电比容量 823.39 和 $373.48 \mathrm{mAh} / \mathrm{g}$ 。

\section{参考文献:}

[1] HU J J, LI G R, GAO X P. Current status, problems and challenges in lithium-sulfur batteries. Journal of Inorganic Materials, 2013, 28(11): $1181-1186$.

[2] YANG Y, ZHENG G Y, CUI Y. Nanostructured sulfur cathodes. Chemical Society Reviews, 2013, 42(7): 3018-3032.

[3] BRUCE P G, FREUNBERGER S A, HARDWICK L J, et al. Li-O and Li-S batteries with high energy storage. Nature Materials, 2011, 11(1): 19-29.

[4] JI X L, NAZAR L F. Advances in Li-S batteries. Journal of Materials Chemistry, 2010, 20(44): 9821-9826.

[5] ZHENG J M, GU M, WANG C M, et al. Controlled nucleation and growth process of $\mathrm{Li}_{2} \mathrm{~S}_{2} / \mathrm{Li}_{2} \mathrm{~S}$ in lithium-sulfur batteries. Journal of the Electrochemical Society, 2013, 160(11): A1992-A1996.

[6] SAH Z W, LI W Y, CHA J J, et al. Sulphur-TiO ${ }_{2}$ yolk-shell nanoarchitecture with internal void space for long-cycle lithiumsulphur batteries. Nature Communications, 2013, 4: 1331-1336.

[7] MIKHAYLIK Y V, AKRIDGE J R. Polysulfide shuttle study in the $\mathrm{Li} / \mathrm{S}$ battery system. Journal of the Electrochemical Society, 2004, 151(11): A1969-A1976.

[8] JI X L, EVERS S, BLACK R, et al. Stabilizing lithium-sulphur cathodes using polysulphide reservoirs. Nature Communications, 2011, 2: 325-331.

[9] KIM M, KANG S H, MANUEL J, et al. Investigation into the role of silica in lithium polysulfide adsorption for lithium sulfur battery. Materials Research Bulletin, 2015, 69: 29-35.

[10] ZHAO X F, HE C Y, LI X L. A novel adsorbent material opal shale. North Environment, 2005, 30(2): 62-63.
[11] YANG D F, WEI C D, NING W K, et al. Structure and adsorption properties of nenjiang opal shale. Journal of Jilin University(Earth Science Edition), 2010, 40(5): 1061-1065.

[12] LI X L, ZHAO X F, ZHANG J L. Study on decolorization of new adsorption material opal shale. North Environment, 2004, 29(1): 37-38.

[13] KIM J H, SEO J, CHOI J, et al. Synergistic ultrathin functional polymer-coated carbon nanotube interlayer for high performance lithium-sulfur batteries. ACS Applied Materials \& Interfaces, 2016, 8(31): 20092-20099.

[14] SUN Q, HE B, ZHANG X Q, et al. Engineering of hollow core-shell interlinked carbon spheres for highly stable lithiumsulfur batteries. ACS Nano, 2015, 9(8): 8504-8513.

[15] WU F, CHEN J Z, CHEN R J, et al. Sulfur/polythiophene with a core/shell structure: synthesis and electrochemical properties of the cathode for rechargeable lithium batteries. The Journal of Physical Chemistry C, 2011, 115(13): 6057-6063.

[16] LI G C, LI G R, YE S H, et al. A polyaniline-coated sulfur/carbon composite with an enhanced high-rate capability as a cathode material for lithium/sulfur batteries. Advanced Energy Materials, 2012, 2(10): 1238-1245.

[17] ZHOU W D, YU Y C, CHEN H, et al. Yolk-shell structure of polyaniline-coated sulfur for lithium-sulfur batteries. Journal of the American Chemical Society, 2013, 135(44): 16736-16743.

[18] GUSTAFSSON G, CAO Y, TREACY G M, et al. Flexible lightemitting diodes made from soluble conducting polymers. Nature, 1992, 357(6378): 477-479.

[19] KRUK M, JARONIEC M. Gas adsorption characterization of ordered organic-inorganic nanocomposite materials. Chemistry of Materials, 2001, 13(10): 3169-3183.

[20] WANG Z D, ZHANG M L, MEI H Y, et al. The physical chemistry explanation of the capillary condensation and the circuit of adsorption-desorption. Xinjiang Petroleum Geology, 2002, 23(3): 233-235.

[21] LI W Y, ZHENG G Y, YANG Y, et al. High-performance hollow sulfur nanostructured battery cathode through a scalable, room temperature, one-step, bottom-up approach. Proceedings of the National Academy of Sciences, 2013, 110(18): 7148-7153.

[22] ATEBAMBA J M, MOSKON J, PEJOVNIK S, et al. On the interpretation of measured impedance spectra of insertion cathodes for lithium-ion batteries. Journal of the Electrochemical Society, 2010, 157(11): A1218-A1228.

[23] CHEN J J, JIA X, SHE Q J, et al. The preparation of nanosulfur/MWCNTs and its electrochemical performance. Electrochimica Acta, 2010, 55(27): 8062-8066. 